\title{
Research Paper: Use of 4.5 Non-locking Plate As an Alternative Device in Open Wedge High Tibial Oste- otomy for Treating Varus Deformity
}

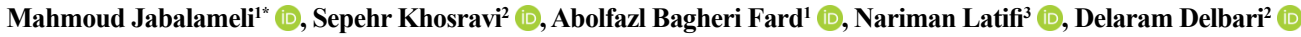 \\ 1. Bone and Joint Reconstruction Research Center, Shafa Orthopedic Hospital, Iran University of Medical Sciences, Tehran, Iran. \\ 2. Student Research Committee, Faculty of Medicine, Iran University of Medical Science, Tehran, Iran. \\ 3. Department of Orthopedic, Shafa Orthopedic Hospital, Iran University of Medical Sciences, Tehran, Iran.
}

\begin{tabular}{|l|l|l}
\hline $\begin{array}{c}\text { Use your device to scan } \\
\text { and read the article online }\end{array}$ & $\begin{array}{l}\text { Crtation Jabalameli M, Khosravi S, Bagheri Fard A, Latifi N, Delbari D. Use of 4.5 Non-locking Plate As an Alternative Device } \\
\text { in Open Wedge High Tibial Osteotomy for Treating Varus Deformity. Journal of Research in Orthopedic Science. 2020; 7(2):55- } \\
\text { 60. http://dx.doi.org/10.32598/JROSJ.7.2.683.1 }\end{array}$ \\
\hline dol: : http://dx.doi.org/10.32598/JROSJ.7.2.683.1
\end{tabular}

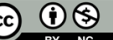

Article info:

Received: 09 Sep 2019

Revised: 18 Dec 2019

Accepted: 2 Feb 2020

Available Online: 01 May 2020

Keywords:

Varus deformity, 4.5 Nonlocking plate, High tibial osteotomy, Open wedge

\section{A B S T RA C T}

Background: High Tibial Osteotomy (HTO) is an approved surgical technique for varus knee deformity. For open wedge HTO multiple fixation methods and devices have been used. Advantages and disadvantages of these methods and devices are reported in various studies. Few studies have been conducted on use and final outcome of correction of varus knee deformity by implementation of non locking plates and benefits of this method is not fully evaluated.

Objectives: To assess clinical and radiographic features of non-locking 4.5 millimeter L-buttress plate and T-buttress plate, which is used in open wedge High Tibial Osteotomy (HTO), and to find out whether this device is efficient enough or not.

Methods: This cross-sectional study was conducted on 39 patients with tibial deformity recruited from a referral orthopedic hospital in Iran. Patients' information, including their baseline characteristics, Range of Motion (ROM) of the knee, comorbidities, time of weight-bearing, union time in X-ray, graft type, and time of follow-up were reported at two stages: before and after the operation. Radiographic images were taken from their legs in both stages. Although different surgeons operated on the cases, they all used the same method. After the surgery, they were checked up in 2, 6, 12, and 24 weeks and a second alignment view was taken from the patients.

Results: Thirty-nine patients underwent surgery for the correction of genu varus deformity and the follow-up time was between 6-48 months. There was no case of non-union and the ROM was perfectly restored in all the patients.

Conclusion: Although the rate of the success in the use of the locking plate in HTO is higher, the use of a 4.5 -mm non-locking plate seems to have decent results, too; therefore, it could be used as an alternative yet functional fixation tool in HTO.

\section{* Corresponding Author:}

Mahmoud Jabalameli, MD.

Address: Bone and Joint Reconstruction Research Center, Shafa Orthopedic Hospital, Iran University of Medical Sciences, Tehran, Iran. Phone: +98 (21) 88758884

E-mail:jabalameli.m@iums.ac.ir 


\section{Introduction}

rom a biomechanical view, varus knee deformity is defined as an excessive load on F the medial compartment [1]. In the case of varus knee, High Tibial Osteotomy (HTO) is an appropriate surgical technique for arthrosis of the knee in adolescents and it is used for cosmetic reasons [2]. HTO is used to shift the load from the degenerative medial compartment to the uninvolved lateral one and curing its damaged articular cartilage.

There are two different methods used for HTO: open and closed wedge osteotomy. Open wedge osteotomy is a technique in which there is no leg shortening or muscle separation. In closed wedge osteotomy, a bone wedge is removed from the lateral side of the bone, and plates, staples, and a tension band system are used for reconstruction [3]. In this operation, there might be some harm done to the peroneal nerve. Also, the separation of extensor muscles and proximal joint of tibiofibular and shortening of fibula could be the disadvantages of closed wedge osteotomy [2].

For the open wedged HTO fixation, there are many tools such as a lock, wedge (in different size), and locking bolts (which create angular stability) $[4,5]$. In developed countries, more modern and expensive plates are used to fix osteotomy. However, plain plaques are alternatives that are not the surgeon's first choice. Although there is now a general tendency to perform an osteotomy by an open wedge method, a consensus cannot be found among surgeons about the use of a standard device for osteotomy fixation [6].

Various devices are used for the correction of varus deformity and their advantages and disadvantages have been discussed in several studies. The non-locking 4.5 LT shape plate is one of the less-noticed devices. This device is several times cheaper than other fixation devices therefore if the results of its use are acceptable compared with other studies, it could lift some financial burden of the patient. In this study, the clinical and radiographic findings of osteotomies that are fixed using this type of plate are reported.

\section{Methods}

\section{Study setting and participants}

This cross-sectional study was conducted in a referral orthopedic center from 2008-2015. The patients with genu varum who underwent open wedge HTO with 4.5 non-locking plates were enrolled. A clear and complete explanation was given to the patients about the need for surgery. The benefits and complications of using allograft or autograft were explained to the patients, too. Completed written consent was received from all the patients and their identities were protected. They were treated according to the ethical guidelines of medical research.

\section{Data collection and the procedure}

Patients' information which included preoperative (sociodemographic data, Range of Motion [ROM], comorbidities) and postoperative information (post-operative ROM of knees operated on, time of weight-bearing, union time in x-ray, graft type, and time of follow-up) was collected by a questionnaire (Table 1).

Pre-operation radiographic images, including AP (anteroposterior) and LAT (Lateral) alignment view of the lower extremity and also different desired angels (LDFA [Lateral Distal Femoral Angle], MPTA [Medial Proximal Tibial Angle], and MFMT [Mechanical Femur Mechanical Tibial] angle) aligned with the alignment view by the orthopedic surgeon, which were taken from all patients and with the consent of professors of knee service, they were chosen for the surgery.

The patients with varus alignments (tibial deformity) who complained of pain and osteoarthritis symptoms in the medial compartment of the knee underwent surgery. Although these operations were performed by different surgeons, they all used a similar surgical method. The patients had checkups and x-ray (AP, LAT) examination at 2, 6, 12, and 24 weeks after the operation. Also, at least 24 weeks after the operation, a second alignment view was given and the angles were measured and recorded.

Following the surgery, the patients were usually nonweight bearing and started knee ROM during the first two weeks. Those who hadn't regain their ROM during the first 6 weeks were advised to perform Physical Therapy (PT) (there was a possibility of non-cooperation in some patients concerning their socioeconomic status, therefore they were monitored earlier than 6 weeks). Possible complications of this surgery include infection, nerve damage, loss of correction, failure of fixation, non-union, and delayed union [7]. Subsequently, after 6 and 12 weeks, the patients got partial and full weight-bearing. 


\section{Surgical technique}

The surgery was performed in the conventional operating room. First a longitudinal skin incision was made along the medial side of patellar ligament up to the medial aspect of the tibia. The tendons of the medial compartment of thigh are released. Then medial opening wedge osteotomy was performed proximal to the tibial tubercle and wedges of the bone with appropriate wedges removed from either iliac crest or implementation of allograft procedure followed by the osteotomy. The superficial part of the medial collateral ligament was exposed at the level of the osteotomy. With the help of osteotomes, cuts were made at the level of osteotomy and three bone wedges depending on the size was inserted. Fixation then was made by 4.5 LT shape non-locking plaques. Next, the tendons of the medial compartment of the thigh and medial collateral ligament were repaired.
The surgery finished with the approximation of subcutaneous tissue and skin.

\section{Statistical analysis}

All the obtained data were entered into SPSS v. 20 (Chicago, IL, USA). For analyzing qualitative variables, the Chi-square test, and for comparing quantitative data, the t-test was used. In all analytic course of action, a Pvalue less than 0.05 was significant.

\section{Results}

A total of 39 patients underwent surgery for the correction of genu varus deformity with using the non-locking plate. The Mean \pm SD patient's age was $63.31 \pm 29.10$ years. Out of 39 patients, $41 \%$ and $59 \%$ were men and women, respectively. Twenty operations $(51.3 \%)$ were conducted on the right knee and 19 (48.7\%) on the left

Table 1. The Mean $\pm S D$ of time to weight-bearing in weeks by the age group $(\mathrm{P}=0.685)$ and the Mean $\pm \mathrm{SD}$ of time of union in weeks by the age group $(\mathrm{P}=0.90)$

\begin{tabular}{ccccc}
\hline & \multicolumn{2}{c}{ Weight-bearing } & \multicolumn{2}{c}{ Union } \\
\cline { 2 - 4 } Variables & Mean \pm SD & P & Mean \pm SD & P \\
\hline$<25(y)(n=13)$ & $11.53 \pm 1.85$ & & $12.07 \pm 1.11$ & 0.90 \\
$25-40(y)(n=16)$ & $13.12 \pm 6.37$ & 0.685 & $13.18 \pm 9.48$ & $12.50 \pm 5.79$ \\
$>40(y)(n=10)$ & $11.90 \pm 5.60$ & & & \\
\hline
\end{tabular}

Orthopedic Science

Table 2. The Mean $\pm S D$ of time, as well as minimum and maximum time in weeks for union and weight-bearing

\begin{tabular}{cccc}
\hline Variables & Minimum & Maximum & Mean \pm SD \\
\hline WB & 8 & 36 & $12.53 \pm 5.00$ \\
U & 7 & 48 & $12.64 \pm 6.63$ \\
\hline
\end{tabular}

WB: weight bearing; U: union

Orthopedic Science

Table 3. The degree of Mean \pm SD, minimum, and maximum pre-operative and post-operative measurements of MPTA, LDFA, and MTMA

\begin{tabular}{ccccccc}
\hline & \multicolumn{2}{c}{ Minimum } & \multicolumn{2}{c}{ Maximum } & \multicolumn{2}{c}{ Mean \pm SD } \\
\cline { 2 - 6 } Variables & Pre-operative & Postoperative & Pre-operative & Post-operative & Pre-operative & Post-operative \\
\cline { 2 - 6 } & 6 & -3 & 28 & 17 & $14.07 \pm 4.92$ & $4.69 \pm 4.75$ \\
\hline MTMA & 87 & 87 & 98 & 98 & $90.79 \pm 2.64$ & $90.89 \pm 2.65$ \\
\hline LDFA & 65 & 81 & 90 & 98 & $79.17 \pm 5.20$ & $88.61 \pm 3.97$ \\
\hline MPTA & & & & & & Orthopedic Science
\end{tabular}

MPTA: Medial Proximal Tibial Angle; LDFA: Lateral Distal Femoral Angle; MTMA: Medial Tibial Mechanical Angle 
Table 4. The study angles degree obtained in the first visit and six months after the operation (preoperative and postoperative angle differences)

\begin{tabular}{ccc}
\hline Variables & Mean \pm SD & $P$ \\
\hline MTMA & $4.15 \pm 9.38$ & 0.0001 \\
LDFA & $0.88 \pm-0.10$ & 0.47 \\
MPTA & $4.65 \pm-9.43$ & 0.0001 \\
\hline
\end{tabular}

MPTA: Medial Proximal Tibial Angle; LDFA: Lateral Distal Femoral Angle; MTMA: Medial Tibial Mechanical Angle.

Table 5. The Mean $\pm \mathrm{SD}$ of time to weight-bearing in weeks by the type of graft $(\mathrm{P}=0.46)$ and the Mean $\pm \mathrm{SD}$ of time of union in weeks by the type of graft $(\mathrm{P}=0.40)$. Also, the average difference of time to weight-bearing by sex $(\mathrm{P}=0.98)$ and the average time of union in weeks by sex $(\mathrm{P}=0.57)$

\begin{tabular}{ccccc}
\hline \multirow{2}{*}{ Variables } & \multicolumn{2}{c}{ Weight-bearing } & Union \\
\cline { 2 - 5 } & Mean \pm SD & $\mathbf{P}$ & Mean \pm SD & P \\
\hline Autograft & $12.17 \pm 3.46$ & 0.46 & $12.17 \pm 3.46$ & 0.40 \\
Allograft & $14 \pm 12.08$ & & $14 \pm 12.08$ & 0.57 \\
Male & $12.13 \pm 3.49$ & 0.98 & $13.37 \pm 9.45$ & $12.13 \pm 3.80$ \\
Female & $13.70 \pm 8.12$ & - & & Orthopedic Science
\end{tabular}

Table 6. The percentages of over and under correction of MPTA after six months of surgery

\begin{tabular}{cc}
\hline MPTA & No. (\%) \\
\hline Over correction $>90^{\circ}$ & $14(35.9)$ \\
\hline Under correction $<84^{\circ}$ & $6(15.4)$ \\
\hline
\end{tabular}

knee. The autograph was the choice for $74.4 \%$ of the patients and allograft for $25.6 \%$. In the follow-up, there was no case of non-union, however, $3(7.7 \%)$ were of delayed union. In 2 of these 3 cases, the union had not occurred until the seventh month after the surgery and for the other patient, this time was up to 12 months after the surgery. ROM for all the patients was perfectly restored except for one who had a reduction of ROM from $0-150$ to $0-140$. All patients were followed up for at least 6 months and at most 48 months. The Mean \pm SD of time for the follow-up was $17 \pm 12.12$ months (Table 1-3).

\section{Discussion}

This study was conducted on 39 patients who underwent open wedge HTO in a referral orthopedic center. The outcomes were measured in MPTA, LDFA, and MTMA during the weight-bearing and union times. The most common complication of HTO is the lack of correction [3]. The non-locking plate is used in this study and there was no report of lack of correction after two years of follow-up. The use of graft in previous operations with a non-locking plate has almost the same success rate with the locking plate [3]. The stability of HTO in genu varus patients depends on the type of plaque used [8-11]. The fact that the patients in this study had successful stability after the operation and during follow-up indicates that non-locking plaque can be used in these patients. In our study, all patients reached union and none manifested any sign of neural or vascular complications during the operation and any device failure or infection after it.

The correction of MPTA and MTMA after the operation compared with the values before the operation were 9.38 and -9.43 , respectively $(\mathrm{P}=0.0001$ for MPTA and $\mathrm{P}=0.0001$ for MTMA). Since these measurements had done six months after the surgery and documented by radiographic consolidation, they do not subject to change in the long term based on previous studies (Table 4-6) [12].

In the patients of this study, $74.4 \%$ had their autografts taken from the iliac crest, and the rest used allografts. The average times of union for autograft and allograft groups were 12.17 and 14 weeks, respectively and since 
the P-value was 0.4 for both autograft and allograft groups there was no difference in implementation of autograft versus allograft in union time post-operation. In a meta-analysis study in 2015, the graft (autogenous iliac bone graft) used in the osteotomy process (autograft, allograft, synthetic material filling, without graft) did not have a major impact on the outcome (the stability and loss of correction) of the surgery (Table 5) [13].

The average times for weight-bearing and union in our study were 12.53 and 12.64 weeks, respectively and these parameters were not influenced by the graft type, sex, and the age group $(\mathrm{P}=0.865$ for weight-bearing regarding age, $\mathrm{P}=0.57$ regarding sex, and $\mathrm{P}=0.46$ regarding the graft type; $\mathrm{P}=0.90$ for the union regarding age, $\mathrm{P}=0.057$ regarding sex, and $\mathrm{P}=0.40$ regarding the graft type) (Table 5).

Our study has shown better results compared with other similar studies such as Rodrigo Salim et al. (2016) who used a similar conventional plate to our study [14]. In the mentioned study, the patients had not reached the partial weight-bearing level even before the eighth week and in our study most patients regain full weight-bearing in about 12 weeks. In their study, the implementation of conventional plates in all patients resulted in radiographic and clinical union within the average time of 16 weeks and the final angles as a measure of the outcome of the operation remained the same after the operation [14].

In Saito et al. study on 77 patients, the average time for the start of full weight-bearing was 18.7 days [15]. In this study, a 5 to 10 years follow-up was done in patients with locking plates who underwent medial high tibia open wedge osteotomy [15].

In another study by Stabli et al. (2011) entitled "open wedge high tibial osteotomy with a locking plate (TomoFix) for the treatment of medial monocomponent osteoarthritis", the ability of weight-bearing was fully regained after the sixth week [11].

These results showed that the average time for people who had used the locking plate was much less than our study [11]. Bone resorption at the site of operation is reported to be a complication of HTO with a non-locking plate $[16,17]$ while this study does not examine this effect in either short or long term. Lateral cortical fracture is a common etiology for loss of correction after surgery in HTO $[18,19]$, and the report indicates a $3 \%$ more probability in the incidence of lateral cortical fracture in non-locking versus locking plaque in the patients [20]. However, studies show that proper fixation could be achieved by both locking and non-locking plates and lateral cortical fracture will be avoided by propped fixation [21]. A major drawback for locking plates compared to non-locking ones is the irritation caused by locking plates [22, 23]. Regarding the time of weight-bearing, the results have discrepancies and some reported earlier weight-bearing in locking plates compared with non-locking patients while this study found that weightbearing could happen at a time similar to the non-locking patients $[19,24,25]$.

\section{Conclusion}

Although using a locking plate has a high success rate in HTO, the price of these products is high. However, since $4.5-\mathrm{mm}$ non-locking plate seems to have decent outcomes with much less price it could be used as an alternative yet functional fixation tool.

\section{Ethical Considerations}

\section{Compliance with ethical guidelines}

The study was approved by the Ethics Committee of Iran University of Medical Sciences.

\section{Funding}

This research did not receive any specific grant from funding agencies in the public, commercial, or non-profit sectors.

\section{Authors' contributions}

Conceptualization, Resources, and Supervision: Mahmoud Jabalameli and Abolfazl Bagheri Fard; Methodology: Mahmoud Jabalameli, Abolfazl Bagheri Fard, and Nariman Latifi; Investigation, Writing - Review \& Editing, and Writing - Original Draft: Sepehr Khosravi, Delaram Delbari, and Nariman Latifi.

\section{Conflict of interest}

The authors declared no conflict of interest.

\section{References}

[1] Staubli AE, Jacob HA. Evolution of open-wedge high-tibial osteotomy: Experience with a special angular stable device for internal fixation without interposition material. Int Orthop. 2010; 34(2):167-72. [DOI:10.1007/s00264-009-0902-2] [PMID] [PMCID] 
[2] LeeSC, Jung KA, Nam CH, Jung SH, Hwang SH. The short-term follow-up results of open wedge high tibial osteotomy with using an Aescula open wedge plate and an allogenic bone graft: The minimum 1-year follow-up results. Clin Orthop Surg. 2010 2(1):47-54. [DOI:10.4055/cios.2010.2.1.47] [PMID] [PMCID]

[3] Agarwala S, Sobti A, Naik S, Chaudhari S. Comparison of closing-wedge and opening-wedge high tibial osteotomies for medial compartment osteoarthritis of knee in Asian population: Mid-term follow-up. J Clin Orthop Trauma. 2016; 7(4):272-5. [DOI:10.1016/j.jcot.2016.06.012] [PMID] [PMCID]

[4] Loia MC, Vanni S, Rosso F, Bonasia DE, Bruzzone M, Dettoni F, et al. High tibial osteotomy in varus knees: Indications and Limits. Joints. 2016; 4(2):98-110. [DOI:10.11138/ jts/2016.4.2.098] [PMID] [PMCID]

[5] Petersen W, Wall A, Paulin T, Park HU, Heymann L. Stability of two angular stable locking plates for open wedge high tibial osteotomy (HTO): TomoFix versus LOQTEQ(R) HTO plate. Arch Orthop Trauma Surg. 2014; 134(10):143742. [DOI:10.1007/s00402-014-2042-4] [PMID]

[6] Pornrattanamaneewong C, Harnroongroj T, Chareancholvanich $\mathrm{K}$. Loss of correction after medial opening wedge high tibial osteotomy: A comparison of locking plates without bone grafts and non-locking compression plates with bone grafts. J Med Assoc Thai. 2012; 95(Suppl. 9):S21-8. [PMID]

[7] Seo S-S, Kim O-G, Seo J-H, Kim D-H, Kim Y-G, Lee I-S. Complications and short-term outcomes of medial opening wedge high tibial osteotomy using a locking plate for medial osteoarthritis of the knee. Knee Surg Relat Res. 2016; 28(4):289-96. [DOI:10.5792/ksrr.16.028] [PMID] [PMCID]

[8] Agneskirchner JD, Freiling D, Hurschler C, Lobenhoffer P. Primary stability of four different implants for opening wedge high tibial osteotomy. Knee Surg Sports Traumatol Arthrosc. 2006; 14(3):291-300. [DOI:10.1007/s00167-005-0690-1] [PMID]

[9] Ribeiro CH, Severino NR, Cury Rde P, de Oliveira VM, Avakian R, Ayhara T, et al. A new fixation material for open-wedge tibial osteotomy for genu varum. Knee. 2009; 16(5):366-70. [DOI:10.1016/j.knee.2009.01.007] [PMID]

[10] Staubli AE. Open-wedge high tibial osteotomy with a locking plate (TomoFix) for treatment of medial monocompartment osteoarthritis. Oper Tech Orthop. 2011; 21(2):156-62 [DOI:10.1053/j.oto.2011.01.004]

[11] Staubli AE, De Simoni C, Babst R, Lobenhoffer P. TomoFix: A new LCP-concept for open wedge osteotomy of the medial proximal tibia--early results in 92 cases. Injury. 2003; 34(Suppl. 2):B55-62. [DOI:10.1016/j.injury.2003.09.025] [PMID]

[12] Sabzevari S, Ebrahimpour A, Roudi MK, Kachooei AR. High tibial osteotomy: A systematic review and current concept. Arch Bone Jt Surg. 2016; 4(3):204-12.

[13] Han JH, Kim HJ, Song JG, Yang JH, Bhandare NN, Fernandez $\mathrm{AR}$, et al. Is bone grafting necessary in opening wedge high tibial osteotomy? A meta-analysis of radiological outcomes. Knee Surg Relat Res. 2015; 27(4):207-20. [DOI:10.5792/ksrr.2015.27.4.207] [PMID] [PMCID]

[14] Salim R, Fogagnolo F, Perina MM, Rubio UM, Kfuri Junior M. Conventional plate and screws in medial openingwedge high tibial osteotomy: Are they sufficiently stable? A retrospective study. Rev Bras Ortop. 2017; 52(5):549-54. [DOI:10.1016/j.rbo.2016.09.008] [PMID] [PMCID]
[15] Saito T, Kumagai K, Akamatsu Y, Kobayashi H, Kusayama Y. Five-to-ten-year outcome following medial opening-wedge high tibial osteotomy with rigid plate fixation in combination with an artificial bone substitute. Bone Joint J. 2014; 96B(3):339-44. [DOI:10.1302/0301-620X.96B3.32525] [PMID]

[16] Getgood A, Collins B, Slynarski K, Kurowska E, Parker D, Engebretsen L, et al. Short-term safety and efficacy of a novel high tibial osteotomy system: A case controlled study. Knee Surg Sports Traumatol Arthrosc. 2013; 21(1):260-9. [DOI:10.1007/s00167-011-1709-4] [PMID]

[17] Han JH, Kim HJ, Song JG, Yang JH, Nakamura R, Shah D, et al. Locking plate versus non-locking plate in open-wedge high tibial osteotomy: A meta-analysis. Knee Surg Sports Traumatol Arthrosc. 2017; 25(3):808-16. [DOI:10.1007/ s00167-015-3850-y] [PMID]

[18] Miller BS, Dorsey WOP, Bryant CR, Austin JC. The effect of lateral cortex disruption and repair on the stability of the medial opening wedge high tibial osteotomy. Am J Sports Med. 2005; 33(10):1552-7. [DOI:10.1177/0363546505275488] [PMID]

[19] Jung WH, Chun CW, Lee JH, Ha JH, Kim JH, Jeong JH. Comparative study of medial opening-wedge high tibial osteotomy using 2 different implants. 2013; 29(6):1063-71. [DOI:10.1016/j.arthro.2013.02.020] [PMID]

[20] Maffulli N, Loppini M, Longo UG, Denaro V, Oliva F. Bovine xenograft locking Puddu plate versus tricalcium phosphate spacer non-locking Puddu plate in opening-wedge high tibial osteotomy: A prospective double-cohort study. Int Orthop. 2013; 37(5):819-26. [DOI:10.1007/s00264-0131817-5] [PMID] [PMCID]

[21] van Raaij TM, Brouwer RW, de Vlieger R, Reijman M, Verhaar JA. Opposite cortical fracture in high tibial osteotomy: Lateral closing compared to the medial openingwedge technique. Acta Orthopaedica. 2008; 79(4):508-14. [DOI:10.1080/17453670710015508] [PMID]

[22] Aryee S, Imhoff AB, Rose T, Tischer T. Do we need synthetic osteotomy augmentation materials for opening-wedge high tibial osteotomy. Biomaterials. 2008; 29(26):3497-502. [DOI:10.1016/j.biomaterials.2008.05.027] [PMID]

[23] Niemeyer P, Schmal H, Hauschild O, von Heyden J, Sudkamp NP, Kostler W. Open-wedge Osteotomy Using an Internal Plate Fixator in Patients With Medial-Compartment Gonarthritis and Varus Malalignment: 3-year Results With Regard to Preoperative Arthroscopic and Radiographic Findings. Arthroscopy. 2010; 26(12):1607-16. [DOI:10.1016/j. arthro.2010.05.006] [PMID]

[24] Hernigou P, Queinnec S, Picard L, Guissou I, Naanaa T, Duffiet $\mathrm{P}$, et al. Safety of a novel high tibial osteotomy locked plate fixation for immediate full weight-bearing: A case-control study. International Orthopaedics. 2013; 37(12):2377-84. [DOI:10.1007/s00264-013-2066-3] [PMID] [PMCID]

[25] Kim MK, Ha JK, Lee DW, Nam SW, Kim JG, Lee YS. No correction angle loss with stable plates in open-wedge high tibial osteotomy. Knee Surgery, Sports Traumatology, Arthroscopy : Official Journal of the ESSKA. 2015; 23(7):19992006. [DOI:10.1007/s00167-014-2842-7] [PMID] 